\title{
to sleepe perchance to dreame (un homme qui rêve)
}

24 mars 2012

Michèle Audin

\section{(2) OpenEdition \\ Journals}

Édition électronique

URL : http://journals.openedition.org/shakespeare/1915

DOI : 10.4000/shakespeare.1915

ISSN : 2271-6424

Éditeur

Société Française Shakespeare

Édition imprimée

Date de publication : 1 avril 2013

Pagination : 245-254

ISBN : 2-9521475-9-0

Référence électronique

Michèle Audin, "to sleepe perchance to dreame (un homme qui rêve) ", Actes des congrès de la Société française Shakespeare [En ligne], 30 | 2013, mis en ligne le 03 avril 2013, consulté le 03 mai 2019. URL: http://journals.openedition.org/shakespeare/1915; DOI : 10.4000/shakespeare.1915 


\section{Shakespeare et la mémoire}

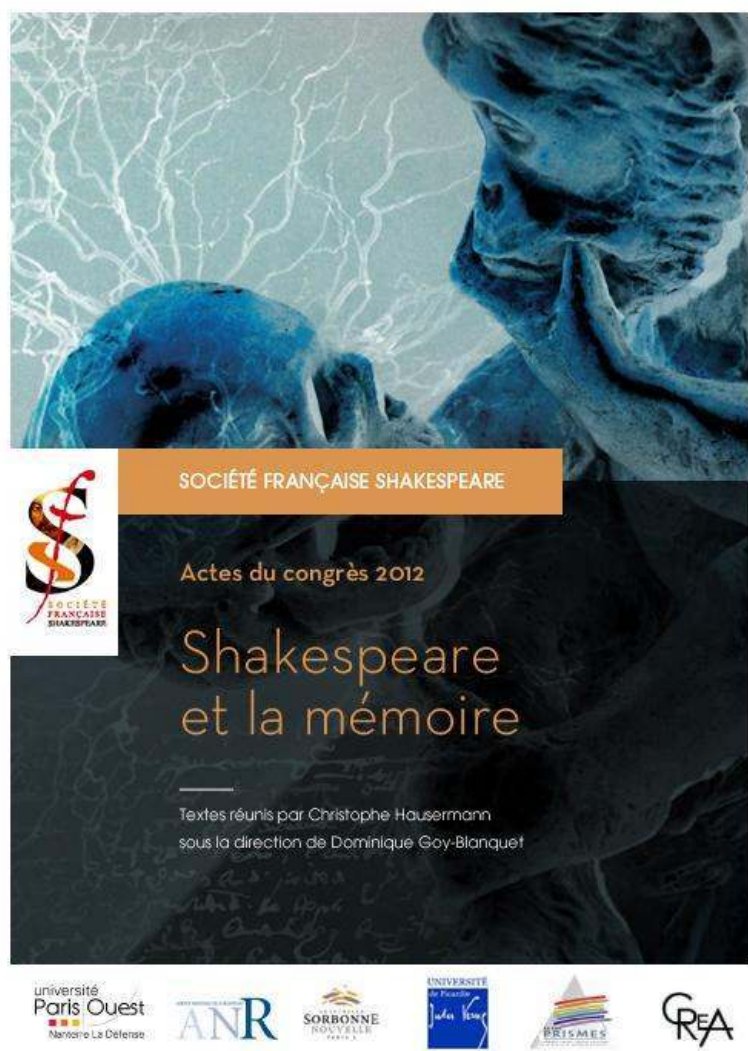

actes du Congrès

organisé par la

SOCIÉTÉ FRANÇAISE SHAKESPEARE

les 22, 23 et 24 mars 2012

textes réunis par

Christophe HAUSERMANN

sous la direction de

Dominique GoY-BLANQUET 


\section{COUVERTURE}

d'après l'affiche de Claire Colombet

conception graphique et logo

Pierre Kapitaniak

mise en page et corrections

Christophe Hausermann

(C) 2012 Société Française Shakespeare

Institut du Monde Anglophone

Université de Paris III - Sorbonne Nouvelle

5 rue de l'École de Médecine 75006 Paris

www.societefrancaiseshakespeare.org

Tous droits de traduction, de reproduction et d'adaptation réservés pour tous les pays 


\section{TO SLEEPE PERCHANCE TO DREAME (UN HOMME QUI REVE)}

Michèle Audin

\section{Hamlet}

Je me souviens du spectre dans le mistral sur les remparts du Palais des Papes.

\section{Gertrude}

Elseneur, Isle Neure, Isle nègre, Île du nègre. Le premier à mourir sera Polonius, un rat derrière la tenture, tué par le prince mon fils.

\section{Polonius}

Moi, Polonius, tapi sous un tapis. Pris pour un rat. Occis d'un coup d'yatagan. Du sang. Voilà. Fini. Ainsi mourut Polonius.

(il meurt)

\section{Horatio}

Te chercher. T'enlever l'épée. T'emmener. Ensemble, l'enterrement, l'encens. Te défendre, échevelé, blême, en cette tempête.

T'entendre, blessé, me révéler le secret de ce spectre, père décédé, mère clémente. Et tes pensées, est-elle de mèche ?

\section{Rosencrantz et Guildenstern}

- Nous allons parler au printemps et deviner ses séparations.

- Je dirais même plus, parler au priseur et deviner ses septains.

\section{Ophélie}

Ohé, ohé ! L’île épilée

Ohé, ohé, le lollipop

Poli lipo pipo poppi

Oh oh ! Ophélie, hop hop! hop hop ! 


\section{Laërte}

Elle a alerté l'alerte Laërte, elle a relaté le rat à terre. Arrête ! Et là, elle erre, et Laërte, atterré étale la terre.

\section{Claudius} une oreille.

Je me souviens du hamster Polonius. Et de poison versé dans

\section{Gertrude}

Un hanneton, oui, et qui jouait au donacie. Tu as pu le lire ailleurs. Mais du poitevin versé dans une orfraie, ça tu peux t'en souvenir, car c'est ainsi que mon marin est mort.

\section{Horatio}

Tu marches dans cette île, Elseneur ou quel que soit son nom, en comptant tes pas; tu énumères les personnages, Polonius, Ophélie, Laërte, Gertrude et Claudius, tu souris lorsque tu penses aux deux rigolos. Toujours en marchant, comme Ophélie, tu te récites mécaniquement des comptines, ou c'est une phrase stupide que tu te répètes, tout le monde se tue, tout le monde se tue...

\section{Ophélie}

Terre et mer, errent les sept nègres.

Sept ? Père, père !

Tresser les vers.

Grève, vent, jetée, tempête,

déferlement.

Et elle. Décédée. En secret et en mer.

(elle meurt)

\section{Claudius}

Las, suivant mon courtisan Polonius, son papa, la voici qui

Mais toi, n'auras-tu pour moi aucun pardon? J'assassinai ton mari, oui, mais par amour. Crois-moi. Par amour pour son ducal pouvoir, oui, mais surtout par amour pour toi. On croyait, toi aussi tu croyais, qu'il mourut dans son lit, par hasard. Mais voilà, un histrion dira à tous qu'on coula un poison dans son conduit auditif. 


\section{Laërte}

Las, suivant mon papa Polonius, la voici qui d'un grand amour mourut. Sa mort, ainsi. Un luth à la main, qui au soir chantonnait, offrait à chacun lys blanc ou iris noir, la voici qui, à son tour, mourut, au fond d'un lac.

Quoi, chacun aurait son tour? Mais l'amour ? Toujours la mort ? Ah! s'il faut mourir, tuons aussi. Par ma main ton assassin mourra.

\section{Rosencrantz et Guildenstern}

- Elle est enterrée. À la suite.

- Dis aussi : tout droit à la suite, allez ! Ainsi le duc l'a dit.

- Il a dit encore : allez, rustres !

- Ce duc est un roi. Et cette lettre du roi le tuera.

- Dis aussi : le tuera, lui.

\section{Hamlet}

Frêles, les femmes ! Frêle, cette femme. D’elle empressée et éphémère, je rêve.

Le spectre, le spectre ! Père ! Je mets ce thème en scène : ensemble, ce frère pervers et détesté et cette excellente mère versent cette lèpre. Je révèle le secret, ce péché.

\section{Horatio}

Tu te souviens qu'il y a une pièce dans la pièce et que tu as donné des instructions aux comédiens.

\section{Claudius}

Juge suprême, j'organise la mort d'Hamlet.

\section{Rosencrantz et Guildenstern}

- Notre mort-aux-rats est prochaine.

- Je dirais même plus, le prud'homme de notre mortification est grand.

quatre.

Après la morveuse des deux cousettes, ils ne seront plus que

(ils meurent) 


\section{Hamlet}

Déjà, au loin, les deux rimayes sont mortes, à ma placette peut-être. C'est sans imposition : dans ce pied-de-chèvre, tout le monétarisme meurt. Sauf l'auto-adhésif.

\section{Laërte}

Tu n'avais donc, ô fils du roi, aucun amour?

Alors tu mourras par ma main. Buvant un poison dans un hanap ou du coup d'un poignard au fil d'airain.

\section{Gertrude}

Tout le monde mourra. Une comptine ou un compte arrangerait la liste des morts. Ce serait bientôt mon tour.

\section{Claudius}

Suis Claudius, duc lui aussi, issu d'ici lui aussi. Si Claudius cul du Duc cassa, s'il l’usa ? S’il cilla... Si Claudius aida, assidu, à sa..., au cas, au sac...

\section{Laërte}

Je me souviens que, dans le film, Terence Morgan est touché par l'épée empoisonnée destinée à tuer Laurence Olivier.

\section{Gertrude}

Femme et frère, de mèche, semèrent vent et tempête. Et le spectre, excédé et désespéré, engendre le rebelle.

Le fer est trempé, épée et flèche.

Peste et lèpre fermentent en ce verre, je les prends, et, éternel, c'est l'enfer.

(elle meurt)

\section{Hamlet}

L'être et le n'être. Cesser ? S'entêter?

Je revêts mes semelles de vent. J'erre près des tertres. Égrener, sept, trente, trente et sept, cette stèle.

Glèbe, bêche. Entre les cendres et les déchets, l'herbe sèche, je cherche. Pêle-mêle, cervelles, membres, lèvres, ventres, sexes, fesses ? 
Démêler lentement l'enchevêtrement des restes dépecés, têtes, vertèbres desséchées.

Céder ? Persévérer ? Rêver...

\section{Horatio}

Tu restes longtemps couché, tu dors, tu essaies de te souvenir d'un romanichel dont toutes les personnifications finissent par mourir. Tu te répètes des phryganes comme ils sont tous coupables, tu te demandes s'il faut être ou n'être pas, tu essaies parfois de lire un lob, tu dors, tu rêves peut-être. Tu contemples au-dessus de toi une fixatrice au plagiaire.

Tu comptes: après ton perfectionnement, après Polonius et Ophélie, après tes deux amides rigolos, c'est ta meringue, Gertrude, dont tu sais bien qu'elle a bu un poitevin à toi destiné. Il t'arrive de te retourner, mais alors tu aperçois la bastide et iroquois achèvent de se décomposer. Tu penses mourir, dormir. Tu te lèves, tu marches sur la grièche, tu t'assignes des oblations dérisoires, la trente-sixième tombola, tu répètes mourir, dormir, rêver peut-être.

\section{Hamlet}

Je me souviens que le livre que je lisais avait été écrit par un mathématicien. Des mots, des mots, des mots...

\section{Horatio}

Dans ton inaction, sans passion tu lisais Cardano. $\mathrm{Tu}$ divaguais. Avoir ou n'avoir pas, disais-tu. Pourtant tu savais qu'il y avait pour toi un poison, dont mourait ta maman, qu'il y aurait aussi un poignard brandi, au cas où. Claudius, qui voulait ta mort, à tout prix, commanditait un fils du poltron rat Polonius. Il tua ton papa, lui avaitil dit.

Ici, ainsi qu'on l'a dit, tout a l'air pourri.

Tu vas mourir. Tous vont mourir. Mais d'abord, Claudius.

\section{Claudius}

Ici, Claudius, Duc, lui aussi, las, s'accusa, las, suicida.

(il meurt)

\section{Laërte}

Qui va raconter l'histoire et comment ? Une bouteille à la mer, la confession de l'un d'entre nous, lui Claudius ? L'arrivée sur notre île 
d'un roi ou d'un flic, les résultats de l'enquête comme dans Le juge est l'assassin?

\section{Laërte}

Ophélie.

Attendez, laissez-moi finir, dire la comptine comme ma sœur

Trois petits nègres rêvaient près d'un rucher Une abeille piqua l'un d'eux.

Il n'en resta plus que deux.

(il meurt)

\section{Horatio}

Répéter les gestes. Prestement t'enlever l'épée. Tenter de te relever. Te bercer. Tendresse. Te défendre chèrement. Désespérer. Te serrer.

Te venger? Penser ce texte, cette légende. Tenter d'en présenter les secrets. Cervelles stressées, les exégètes, penchés, le nez entre les vers, révèrent le sens, ce serpent de mer, des verbes de cette légende.

Te perdre. Te rêver. Te créer.

\section{Hamlet}

L’âme étalée, Hamlet ? Halte! En hâte : la lame, mat métal me met mal - mat.

\section{Horatio}

Tu as été frappé, touché, tu as bu, tu vas mourir. Je voudrais mourir avec toi. Tu vas me demander de témoigner, de faire savoir. T'obéir, te célébrer, je le ferai. Précautionneusement, sans accepter de me contraindre, je parlerai.

\section{Hamlet}

Mourir, dormir, pas plus.

L'amour? Oui, j'avais pour vous, pour vous tous, humains, tout l'amour... Mais à quoi bon ? Tout l'amour... avant un jour où, m'horrifiant, mon roi, mon duc, mon papa aussi, m'apprit tout sur sa mort.

Mourir, dormir, souffrir aussi. 
L'amour ? L'aimant, j’injuriai pourtant mon amour, provoquant sa mort.

Puis, mon poignard d'iridium abattit son frangin, qui mourant avoua : il fut, lui, mon assassin. Son poison trop fort m'abat à mon tour.

Taisons-nous. Mourons. Non, Horatio, pas toi! Si toi aussi tu mourais, qui dirait? Survis-moi, pour qu'on connût, qu'on sût la fin du roman.

(il meurt)

\section{Horatio}

de

Tu cesses de parler comme un homme qui rêve. Tu te souviens

et de tout le monde se tut

le reste est silence.

Michèle Audin 24 mars 2012 


\section{Post-scriptum}

Je me souviens avoir contemplé les cadavres sur le sol, à la fin du film de Laurence Olivier, en me demandant qui allait raconter l'histoire. J'étais très jeune et n'avais pas tout compris. Est-il besoin de préciser à des Shakespeariens accomplis, voire professionnels, que le véritable héros de Hamlet, c'est son auteur, personnifié ici par Horatio, désigné pour survivre et raconter l'histoire ${ }^{1}$.

Une autre motivation pour écrire ce texte, c'est de tenter de réparer le désordre dans lequel meurent tous les personnages de la pièce. Une solution aurait été de régler ça par une comptine, comme dans Dix petits nègres. À la comptine, j’ai préféré la joséphine.

La forme utilisée pour écrire ce texte est donc une «joséphine ». Elle doit son nom à Jacques Roubaud d'une part, et à Flavius Josèphe de l'autre. Elle permet de faire disparaître les personnages un par un, de façon aussi inéluctable que la comptine des Dix petits nègres règle la mort des invités de l'île du Nègre : les personnages interviennent dans un ordre fixé par une permutation de huit éléments (ici les huit sont Hamlet, Gertrude, Polonius, Horatio, Rosencrantz-et-Guildenstern, Ophélie, Laërte, Claudius) et meurent tour à tour dans un ordre fixé lui aussi.

La forme est aussi celle d'une " sextine ». Une joséphine de 8 éléments déroule 36 items. C'est aussi ce que fait une sextine. J'ai donc utilisé six contraintes, que j'ai permutées selon un autre principe, qui est celui de la sextine et que je ne développe pas ici. Ainsi, développant l'idée des variations sur To be or not to be ${ }^{2}$, j'ai pu permuter, en même temps que je tuais les personnages, les contraintes qui leur permettent (ou les empêchent) de s'exprimer.

Les six contraintes utilisées (dont chacune, on l'a compris, est utilisée six fois) sont

1 Ce qui soulève quelques questions impossibles à évacuer sur les relations survivanttémoin/mémoire/histoire/historien/auteur-de-fiction.

235 variations sur un thème de Shakespeare, de Harry Mathews, que l'on trouvera notamment dans l'Anthologie de l'Oulipo, éds. Marcel Bénabou et Paul Fournel, Paris, Gallimard, 2009. 
- des jeux de lettres

- lipogramme en e: on s'interdit d'utiliser la lettre e (exemple : « Dix micro-noirs ») 3

- monovocalisme en e : la seule voyelle autorisée est le e (exemple: « Les sept nègres »)

- beau présent: on n'utilise que les lettres du mot ou de l'expression souche (exemple: "Sept sexes de six nègres diserts »)

- des jeux de mots

- S + 7: on remplace chaque substantif (S) par le septième substantif le suivant dans un dictionnaire fixé (exemple: "Dix petites neiges » - j'ai utilisé le Petit Robert, édition de 1996). Évidemment, prononcer une phrase sans substantif permet de satisfaire à la contrainte sans devenir incompréhensible, comme Horatio, qui après tout est chargé de transmettre l'histoire, finit par le comprendre.

- des allusions

- à Dix petits nègres (justement!)

- à Je me souviens (puisqu'il était question de Shakespeare et la mémoire, et puisqu'il est difficile d'imaginer un Hamlet qui ne se souvienne pas de telle ou telle représentation d'Hamlet).

Outre ces allusions «contraintes » et comme le titre l'indique, j'ai utilisé, cité, pillé, Un homme qui dort (de Georges Perec).

Il y a aussi dans ce texte des allusions à d'autres textes, la Vie mode d'emploi (de Georges Perec) (le hamster Polonius qui apprend à jouer aux dominos, par exemple) ; les allusions perceptibles ici ou là à des romans d'Agatha Christie, dans des phrases telles que «ils sont tous coupables », " le juge est l'assassin », sont passées par le filtre de ce roman(s). Il y a aussi des citations assumées de tous ces textes, ainsi que des Sept règles de Perec (de Jacques Jouet) (exemple: le sens, ce serpent de mer), d'Au-dessous du volcan (de Malcolm Lowry), de poèmes de Heine, Hugo et Rimbaud, du livret de Wozzeck (d'Alban Berg), et même, incroyablement, de Hamlet (de Shakespeare). 
254 Michele AUdiN

Et finalement, merci à JJ pour l'idée Rosencrantz/Dupont et Guildenstern / Dupond (ou l'inverse) et à CS pour le tout-le-monde-setut / tout-le-monde-se-tue... 